\title{
(-)-Epigallocatechin-3-O-gallate (EGCG) attenuates the hemodynamics stimulated by caffeine through decrease of catecholamine release
}

\author{
Ki-Wan Oh, Jong-Myeong Hong, Jin Tae Hong
}

\section{Chunbuk National University, Korea}

A human study of the effects of hemodynamics of caffeine and epigallocatechin-3-O-gallate (EGCG) was performed. Caffeine tablets $(200 \mathrm{mg})$ were orally administered to healthy males aged 25 to 35 years 30 min after oral administration of EGCG tablets (100 and $200 \mathrm{mg}$ ). The increase in BP induced by caffeine was inhibited when co-administered with EGCG. We found that caffeine slightly decreased heart rate (HR). Although EGCG enhanced HR reducton. the effect was not significant. In addition, caffeine increased blood catechloamines levels. but EGCG inhibited the increase in noradrenaline, adrenaline and dopmaine levels induced by caffeine. Whether EGCG decreases the elevated HR and systolic perfusion pressure, and ventricualar contractility induced by adrenergic agonists in the isolated rat heart was investigated. The modified Krebs-Henseleit solution was perfused through a Langendorff apparatus to the isolated heart of rats. HR, systolic perfusion pressure, and deveoloped maximal rates of contraction and relaxation were increased by epinephrine (EP) and isoproterenol (IP). In contrast, EGCG decreased the elevated HR, systolic perfusion pressure, and left ventricular pressure induced by EP and/or IP. In confusion, EGCG could attenuate the hemodynamics stimulated by caffeine through decreasing catecholamines release. 